\title{
Transient particle energies in shortcuts to adiabatic expansions of harmonic traps
}

\author{
Yang-Yang Cui, ${ }^{1}$ Xi Chen, ${ }^{1}$ and J. G. Muga ${ }^{2,1}$ \\ ${ }^{1}$ Department of Physics, Shanghai University, 200444 Shanghai, People's Republic of China \\ ${ }^{2}$ Departamento de Química-Física, UPV-EHU, Apdo 644, 48080 Bilbao, Spain
}

\begin{abstract}
The expansion of a harmonic potential that holds a quantum particle may be realized without any final particle excitation but much faster than adiabatically via "shortcuts to adiabaticity" (STA). While ideally the process time can be reduced to zero, practical limitations and constraints impose minimal finite times for the externally controlled time-dependent frequency protocols. We examine the role of different time-averaged energies (total, kinetic, potential, non-adiabatic) and of the instantaneous power in characterizing or selecting different protocols. Specifically, we prove a virial theorem for STA processes, set minimal energies for specific times or viceversa, and discuss their realizability by means of Dirac impulses or otherwise.
\end{abstract}

PACS numbers: 03.75.-b, 37.10.De

\section{INTRODUCTION}

The need to shorten the times of adiabatic process in different quantum or classical systems has motivated a surge of activity to design "Shortcuts to adiabaticity" (STA), namely, fast protocols for the external parameters controlling the system that are generically non-adiabatic but provide the same final populations than the adiabatic dynamics, see [1] and references therein. The timedependent harmonic oscillator is a paradigmatic study case, as it represents generically the conditions near equilibrium and has allowed for experimental implementations 2, 3], and applications in many different classical or quantum systems [4-30]. For anharmonic corrections see as well [31, 32]. Since a basic aim of the shortcuts is to shorten the process time $t_{f}$, some "price" in the form of high transient energies may be expected to be paid. Several articles have studied the energies and protocol times involved: their characterization; their mutual relation, which is not necessarily of the simple form of a time-energy uncertainty principle; and also their optimization under different constraints and conditions [8, 12, 14]. In this paper we build on these results and continue the analysis of the energies and times in STA processes. We do not aim at being comprehensive but at examining points that either had not been discussed or needed some clarification. Our motivation here is of fundamental nature, but the results will be relevant to quantify the third principle of thermodynamics [4, 7, 8], as well as the speed, efficiency limits, and costs of quantum engines and refrigerators [4, 14, 27 29, 33].

After a quick review of invariant-based engineering in the Introduction, we shall first put forward in Sec. II a virial theorem for STA processes which relates timeaveraged total, kinetic and potential energies, $\bar{E}, \bar{K}, \bar{V}$. We then discuss an idealized protocol that minimizes the time-averaged energy for a given time $t_{f}$, or viceversa, by means of Dirac-delta impulses of the spring constant. It is shown that these impulses contribute to the total averaged energy and we specify how much. After a comparison of several protocols in a $\left(\bar{E}, t_{f}\right)$ plane we study the non-adiabatic energy and its time-average in Sec. II. Its lower bound for given $t_{f}$ is found and its implementation is discussed. We also compare different protocols for this quantity. Finally, the instantaneous power in STA processes is examined in Sec. IV] and we end up with a discussion.

\section{A. Invariant-based inverse engineering}

Here we consider a particle of mass $m$ in a harmonic trap, with time-dependent Hamiltonian

$$
H(t)=\underbrace{\frac{p^{2}}{2 m}}_{K}+\underbrace{\frac{1}{2} m \omega(t)^{2} q^{2}}_{V},
$$

where $\omega(t)$ is the time-dependent angular frequency of the trap (from now on "frequency" will be understood as an angular frequency), and $q$ and $p$ are position and momentum operators, with $q$ being just a $c$-number in coordinate representation. Lewis and Riesenfeld found the quadratic invariant [34] $I(t)=\pi^{2} / 2 m+m \omega_{0}^{2} q^{2} / b^{2}$, where $\omega_{0}=\omega(0)$ is the initial frequency, $\pi=b p-m \dot{b} q$ is a momentum conjugate to $q / b$, the dots represent derivative with respect to time, and $b \equiv b(t)$ is a timedependent, dimensionless scaling function proportional to the width of the elementary dynamical modes. Since an invariant $I(t)$ must satisfy $-i[I, H] / \hbar+\partial I / \partial t=0$, so that $\langle\Psi(t)|I(t)| \Psi(t)\rangle$ remains constant for any $|\Psi(t)\rangle$ that evolves with $H(t), b$ must obey the Ermakov equation

$$
\ddot{b}+\omega^{2}(t) b=\frac{\omega_{0}^{2}}{b^{3}} .
$$

Any state evolving with $H(t)$ can be expanded as a superposition of "dynamical modes" $|\Psi(t)\rangle=\sum_{n} a_{n}\left|\psi_{n}(t)\right\rangle$, where $n=0,1,2, .$. , the $a_{n}$ are time-independent amplitudes, $\left|\psi_{n}(t)\right\rangle=e^{i \alpha_{n}(t)}\left|u_{n}(t)\right\rangle, \quad \alpha_{n}(t)=-(n+$ $\left.\frac{1}{2}\right) \omega_{0} \int_{0}^{t} d t^{\prime} / b^{2}\left(t^{\prime}\right)$ are Lewis-Riesenfeld phase factors, and the $\left|u_{n}(t)\right\rangle$ are eigenvectors of the invariant, $I(t)\left|u_{n}(t)\right\rangle=$ $\lambda_{n}\left|u_{n}(t)\right\rangle$, where $\lambda_{n}=(n+1 / 2) \hbar \omega_{0}$ are time-independent eigenvalues. 
The dynamical modes have the form

$$
\begin{aligned}
\left\langle q \mid \psi_{n}(t)\right\rangle & =\left(\frac{m \omega_{0}}{\pi \hbar}\right)^{\frac{1}{4}} \frac{e^{-i(n+1 / 2) \int_{0}^{t}\left(\omega_{0} / b^{2}\right) d t^{\prime}}}{\left(2^{n} n ! b\right)^{1 / 2}} \\
& \times e^{i \frac{m}{2 \hbar}\left(\frac{\dot{b}}{b}+i \frac{\omega_{0}}{b^{2}}\right) q^{2}} \mathcal{H}_{n}\left[\left(\frac{m \omega_{0}}{\hbar}\right)^{\frac{1}{2}} \frac{q}{b}\right],
\end{aligned}
$$

where $\mathcal{H}_{n}$ is a Hermite polynomial. If the commutation relations $[H(0), I(0)]=0$ and $\left[H\left(t_{f}\right), I\left(t_{f}\right)\right]=0$ are satisfied the initial eigenstates of $H$ are mapped dynamically into final eigenstates, avoiding final particle excitation [7]. These commutation relations indeed hold if the boundary conditions

$$
\begin{aligned}
& b(0)=1, \quad \dot{b}(0)=0, \\
& b\left(t_{f}\right)=\gamma, \quad \dot{b}\left(t_{f}\right)=0,
\end{aligned}
$$

are satisfied, where $\gamma=\sqrt{\omega_{0} / \omega_{f}}$, and $\omega_{f}$ is the final frequency. In addition,

$$
\ddot{b}(0)=\ddot{b}\left(t_{f}\right)=0
$$

may be imposed to make $\omega(t)$ continuous at $t=0$ and $t=t_{f}$. To inverse engineer $\omega(t), b(t)$ is designed first, interpolating with some convenient function between the boundary conditions at 0 and $t_{f}$, and $\omega(t)$ is deduced from the Ermakov equation (2) [7]. A simple protocol based on a quintic polynomial for $b(t)$, where the coefficients are found from Eqs. (44) and (5), is 7]

$$
b(t)=6(\gamma-1) s^{5}-15(\gamma-1) s^{4}+10(\gamma-1) s^{3}+1,
$$

where $s=t / t_{f}$. For very short times, imaginary frequencies appear for some intermediate times, corresponding to a negative, concave-down potential.

Another simple solution is the "bang-bang" (stepwise constant) form of the control function $\omega(t)$. It results in particular from applying optimal control theory (OCT) to minimize the time for given constraints on the frequency [4, 7, 11, 14]. Some relevant expressions are provided in the Appendix. As well, "bang-singular-bang" solutions result from minimizing the time-averaged energy for the same constraints [12].

\section{TOTAL, KINETIC AND POTENTIAL ENERGIES}

For the $n$-th dynamical mode, the instantaneous energy, $E_{n}(t) \equiv\left\langle\psi_{n}|H(t)| \psi_{n}\right\rangle$, is given by

$$
E_{n}(t)=\frac{(2 n+1) \hbar}{4 \omega_{0}}\left[\dot{b}^{2}+\omega^{2}(t) b^{2}+\frac{\omega_{0}^{2}}{b^{2}}\right] .
$$

We may divide the energy into kinetic and potential parts using the separation $H=K+V$ in Eq. (11). Then $E_{n}(t)=K_{n}(t)+V_{n}(t)$, where

$$
\begin{aligned}
& K_{n}(t)=\frac{(2 n+1) \hbar}{4 \omega_{0}}\left(\dot{b}^{2}+\frac{\omega_{0}^{2}}{b^{2}}\right), \\
& V_{n}(t)=\frac{(2 n+1) \hbar}{4 \omega_{0}} b^{2} \omega^{2}(t) .
\end{aligned}
$$

The mean value theorem implies for the interval $\left(0, t_{f}\right)$, assuming that the boundary conditions (44) are satisfied [35],

$$
\dot{b}_{\max } \geq \frac{\gamma-1}{t_{f}}, \quad\left|\ddot{b}_{\max }\right| \geq 2 \frac{\gamma-1}{t_{f}^{2}} .
$$

The first inequality sets a lower bound for the maximum of the instantaneous kinetic energy, $K_{n, \max } \geq$ $\frac{(2 n+1) \hbar}{4 \omega_{0}} \frac{(\gamma-1)^{2}}{t_{f}^{2}}$. In general we cannot set bounds for minimal or maximal values of $V_{n}$ unless physically motivated bounds apply for both $b$ and $\omega$.

\section{A. Virial theorem for STA processes}

A virial-theorem relation will be proved for STA processes that implies, remarkably, that the time-averaged energy is equipartitioned into kinetic and potential contributions, namely, $\overline{K_{n}}=\overline{V_{n}}=\overline{E_{n}} / 2$, where the overline denotes a time average over $\left[0, t_{f}\right]$, i.e., for a generic $A_{n}(t)$ function,

$$
\overline{A_{n}}=\frac{1}{t_{f}} \int_{0}^{t_{f}} A_{n}(t) d t
$$

The virial relation for STA processes is not at all an obvious result, as the ordinary quantum-mechanical virial theorem applies to localized (square integrable) stationary waves and a time-independent Hamiltonian [36 38]. Generalizations were proposed for time-dependent states and a time-independent Hamiltonian, performing the average over an infinite time or over a period if the motion is periodic 39 41]. Finally, in Ref. 42 a virial theorem was found for a charged particle in a time-dependent electromagnetic field.

Suppose first that the trap expansion is performed adiabatically over a very long time $t_{f}$ so that the state remains in level $n$ from $t=0$ to $t_{f}$. The ordinary virial theorem, i.e., the one formulated for stationary states, can then be applied. For the harmonic oscillator in level $n$, it states that $V_{n}=K_{n}=E_{n} / 2$. Along the adiabatic process the values of the energies will change slowly in such a way that the relation is preserved. We may thus take a time average and get $\overline{V_{n}}=\overline{K_{n}}=\overline{E_{n}} / 2$. For an arbitrary non-adiabatic process, however, this relation will not be true in general.

According to Eq. (7) and Eq. (11), the time-averaged energy is

$$
\overline{E_{n}}=\frac{1}{t_{f}} \int_{0}^{t_{f}} \frac{(2 n+1) \hbar}{4 \omega_{0}}\left[\dot{b}^{2}+\omega^{2}(t) b^{2}+\frac{\omega_{0}^{2}}{b^{2}}\right] d t
$$

or, using Ermakov's equation (2),

$$
\overline{E_{n}}=\frac{1}{t_{f}} \int_{0}^{t_{f}} \frac{(2 n+1) \hbar}{4 \omega_{0}}\left[2 \frac{\omega_{0}^{2}}{b^{2}}+\dot{b}^{2}-\ddot{b} b\right] d t .
$$


By using the boundary conditions (4) and partial integration, we have $\int_{0}^{t_{f}}(-\ddot{b} b) d t=\int_{0}^{t_{f}} \dot{b}^{2} d t$. Then the timeaveraged energy can be rewritten as, see Eq. (8),

$$
\overline{E_{n}}=\overline{E_{n, 2}} \equiv \frac{1}{t_{f}} \int_{0}^{t_{f}} \frac{(2 n+1) \hbar}{2 \omega_{0}}\left[\frac{\omega_{0}^{2}}{b^{2}}+\dot{b}^{2}\right]=2 \overline{K_{n}} .
$$

(We have introduced a specific notation, $\overline{E_{n, 2}}$ for the integral in Eq. (14) to emphasize that $\overline{E_{n, 2}}$ is only the averaged energy when the boundary conditions are satisfied.) Since $\overline{E_{n}}=\overline{K_{n}}+\overline{V_{n}}$, we find, remarkably, the virial-theorem relation

$$
\overline{K_{n}}=\overline{V_{n}}=\overline{E_{n}} / 2 .
$$

This implies a number of consequences. For example, minimizing any of the time-averaged energies automatically minimizes the others. As well, non-trivial bounds may be set since bounds for one of the averaged energies works for the others as well. Thus, in spite of the fact that $V_{n}$ may be negative during some time interval, the time-average $\overline{V_{n}}$ must be positive, as $K_{n} \geq 0$; even more, it will be bounded from below by a positive number as described in the next subsection. Further applications in this work may be found in Sec. IIIA and in the Appendix.

\section{B. The energy contribution from Dirac impulses}

Using the Euler-Lagrange equation [8] to minimize Eq. (14) or Optimal Control Theory (OCT) [12], the following quasi-optimal function is found,

$$
b(t)=\sqrt{\left(B^{2}-\omega_{0}^{2} t_{f}^{2}\right)\left(\frac{t}{t_{f}}\right)^{2}+2 B\left(\frac{t}{t_{f}}\right)+1},
$$

where $B=\sqrt{\omega_{0}^{2} t_{f}^{2}+\gamma^{2}}-1$ and the positive root should be taken; this only satisfies the boundary conditions $b(0)=1$ and $b\left(t_{f}\right)=\gamma$ at the time limits but not the conditions on the derivatives. Therefore, as such it only provides a bound for the time-averaged energy. Substituting Eq. (16) into Eq. (14), this lower bound is

$$
\begin{aligned}
& \overline{E_{n L}}=\frac{(2 n+1) \hbar}{2 \omega_{0} t_{f}^{2}}\left\{\left(B^{2}-\omega_{0}^{2} t_{f}^{2}\right)-2 \omega_{0} t_{f}\right. \\
& \left.\times\left[\operatorname{arctanh}\left(\frac{B^{2}+B-\omega_{0}^{2} t_{f}^{2}}{\omega_{0} t_{f}}\right)-\operatorname{arctanh}\left(\frac{B}{\omega_{0} t_{f}}\right)\right]\right\},
\end{aligned}
$$

which becomes $\overline{E_{n L}} \approx \frac{(2 n+1) \hbar}{2 \omega_{f} t_{f}^{2}}$ for $t_{f} \ll 1 / \sqrt{\omega_{0} \omega_{f}}$ and $\gamma \gg 1$. In [8], polynomial "caps" were added around a central time-segment defined by Eq. (16), to match this function with the right boundary conditions. A numerical example for $t_{f} \ll 1 / \sqrt{\omega_{0} \omega_{f}}$ and $\gamma \gg 1$ showed that when the cap duration $\tau$ went to zero, $\overline{E_{n}} \rightarrow \overline{E_{n L}}$. However the caps did contribute to the integral, actually half of the total time-averaged energy came from them, but the significance of this fact was not discussed.

In [12] an alternative to the polynomial caps was put forward, namely, Dirac-delta impulses of the control function $\omega^{2}(t)$ that switch the derivatives $\dot{b}\left(0^{+}\right)$and $\dot{b}\left(t_{f}^{-}\right)$to $\dot{b}\left(0^{-}\right)=\dot{b}\left(t_{f}^{+}\right)=0$,

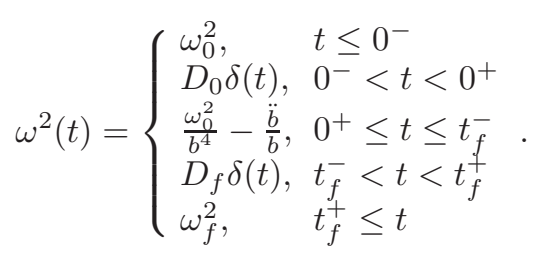

Substituting the $\delta$-terms in the Ermakov equation (2), integrating $\ddot{b}$ around (in the immediate neighborhood of) $t=0$, and $t_{f}$, and taking into account the boundary conditions in (4), $D_{0}$ and $D_{f}$ are found to be

$$
D_{0}=-\frac{\dot{b}\left(0^{+}\right)}{b(0)}, D_{f}=\frac{\dot{b}\left(t_{f}^{-}\right)}{b\left(t_{f}\right)} .
$$

(The first impulse always corresponds to a negative Delta, while the second may have the two signs [12].) The protocol (18) with Dirac impulses provides a formally elegant proof that the bound can indeed be reached, at least in principle, because, as the boundary conditions are satisfied, Eq. (14) may be used to get Eq. (17) as the actual time-averaged energy of the process. Note that these protocols minimize the time-averaged energy for a given time $t_{f}$, but also minimize the time $t_{f}$ for a given time-averaged energy. In the idealized processes that realize the bound the instantaneous potential energy jumps to (plus or minus) infinity due to the Dirac pulses. It might seem that the deltas do not contribute to the averaged energy since $\omega$ does not appear explicitly in Eq. (14). However they do. To see why and how much, the time-averaged energy given by the original expression, Eq. (12), where no partial integration has been carried out, can be separated into two parts, one from the deltas and one from the central time interval,

$$
\overline{E_{n}}=\Delta_{\delta}+\overline{E_{n}}\left[0^{+}, t_{f}^{-}\right],
$$

where

$$
\begin{aligned}
& \Delta_{\delta}=\int_{0^{-}}^{0^{+}} E_{n}(t) d t+\int_{t_{f}^{-}}^{t_{f}^{+}} E_{n}(t) d t, \\
& \overline{E_{n}}\left[0^{+}, t_{f}^{-}\right]=\int_{0^{+}}^{t_{f}^{-}} E_{n}(t) d t
\end{aligned}
$$

and $E_{n}(t)$ is given in Eq. (77) with the frequencies in Eq. (18). Making use of the coefficients (19), the contribution from the Dirac impulses to the time-averaged energy is

$$
\Delta_{\delta}=\frac{(2 n+1) \hbar}{4 \omega_{0} t_{f}}\left[\dot{b}\left(t_{f}^{-}\right) b\left(t_{f}\right)-\dot{b}\left(0^{+}\right) b(0)\right] .
$$


The other term in Eq. (20) can be rewritten using partial integration as

$$
\overline{E_{n}}\left[0^{+}, t_{f}^{-}\right]=\overline{E_{n, 2}}+\Delta_{\text {boundary }},
$$

where

$$
\Delta_{\text {boundary }}=-\frac{(2 n+1) \hbar}{4 \omega_{0} t_{f}}\left[\dot{b}\left(t_{f}^{-}\right) b\left(t_{f}\right)-\dot{b}\left(0^{+}\right) b(0)\right] .
$$

Thus it turns out that

$$
\Delta_{\delta}=-\Delta_{\text {boundary }}
$$

so, according to Eqs. (20, 24, 26),

$$
\overline{E_{n}}=\overline{E_{n, 2}}=\overline{E_{n L}} \text {. }
$$

Substituting the quasi-optimal protocol (16) in Eq. (23),

$$
\Delta_{\delta}=\frac{(2 n+1) \hbar}{4 \omega_{0} t_{f}^{2}}\left(B^{2}-\omega_{0}^{2} t_{f}^{2}\right) .
$$

When $t_{f} \ll 1 / \sqrt{\omega_{0} \omega_{f}}$ and $\gamma \gg 1$,

$$
\Delta_{\delta} \approx \frac{(2 n+1) \hbar}{4 \omega_{f} t_{f}^{2}}=\overline{E_{n L}} / 2,
$$

which agrees with the contribution of the caps found numerically in [8] as $\tau \rightarrow 0$. The reason for this result did however escape the authors of $[8]$.

\section{Comparison of protocols}

Figure 1 depicts $t_{f}$ versus the time-averaged energy for different protocols. A log-log representation is chosen to show the global behavior and the domains where the protocols are applicable. As announced the fastest protocols for a given $\overline{E_{n}}$ are the ones that implement the bound (17). Other constraints lead to different winners. For example, if $\left|\omega^{2}\right|$ is limited by some predetermined value the fastest solutions are of bang-bang form [7, 14]. If in addition to this bound the time-averaged energy is fixed, they are of bang-singular-bang form [12], where the protocol in Eq. (16) applies in a central segment flanked by constant-frequency intervals. The bang-bang protocol considered in Fig. 1 assumes the two intermediate frequency steps $i \omega_{1}$ and $\omega_{1}$ for durations $t_{1}$ and $t_{2}$, respectively; $\omega_{1}$ decreases for larger times, and a maximal time (minimal $\overline{E_{n}}$ ) exists, as discussed in the Appendix and shown in the figure.

\section{NON-ADIABATIC ENERGY}

The "non-adiabatic energy" is defined as the difference between the total energy and the energy of a corresponding adiabatic process,

$$
\begin{aligned}
E^{n a}(t) & =\langle H(t)\rangle-E_{a d}(t) \\
& =\sum_{j}\left[\mathcal{P}_{j}(t)-\mathcal{P}_{j}(0)\right] \epsilon_{j}(t),
\end{aligned}
$$

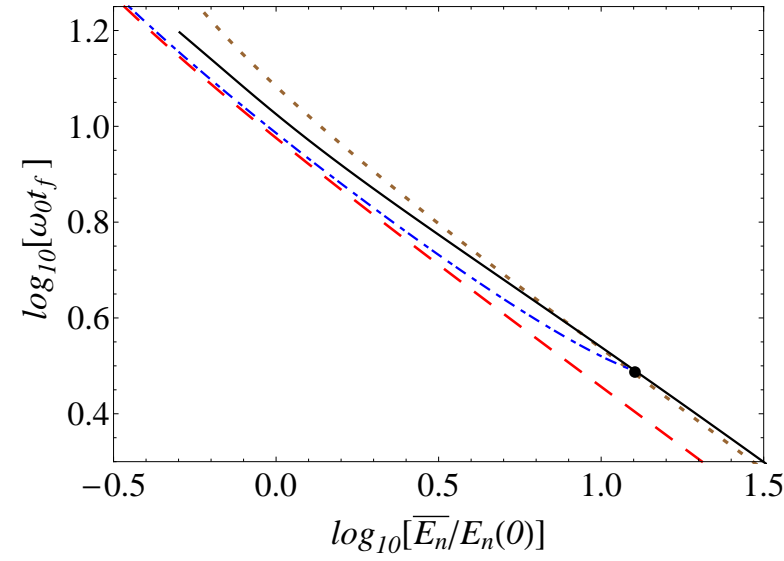

FIG. 1: (Color online) Protocol time $t_{f}$ versus time-averaged energy $\overline{E_{n}}$ for a quintic polynomial protocol (dotted brown), bang-bang for $\omega_{1}=\omega_{2}$, which decreases for larger times, see Eq. A1 (solid black), and lower bound (dashed red). The filled black point is for the bang-bang corresponding to $-\omega_{0}^{2} \leq$ $\omega^{2}(t) \leq \omega_{0}^{2}$. The bang-singular-bang form (dot-dashed blue) is for the same bounds, with the bang-bang control of the point as its limiting, minimal-time case. Parameters: $\omega_{0}=$ $2500 \times 2 \pi \mathrm{Hz}$ and $\omega_{f}=25 \times 2 \pi \mathrm{Hz}$.

where the $\epsilon_{j}(t)=(j+1 / 2) \hbar \omega(t)$ are the instantaneous eigenenergies, the $\mathcal{P}_{j}(t)$ the corresponding populations, and $E_{a d} \equiv \sum_{j} \mathcal{P}_{j}(0) \epsilon_{j}(t)$. We must now assume $\omega(t) \geq 0$ in order to have a meaningful real quantity. The "minimal work principle" [43], establishes that $\langle H(t)\rangle \geq E_{a d}(t)$ provided that the initial state is passive (i.e., the initial density matrix is diagonal in the energy representation and satisfies $\left.\mathcal{P}_{n}(0) \geq \mathcal{P}_{n+1}(0)\right)$ and no level-crossings occur. We shall restrict this section to the ground state $\left|\psi_{0}\right\rangle$ as a simple passive state. Then, from Eq. (7),

$$
E^{n a}(t)=\frac{\hbar}{4 \omega_{0}}\left(\dot{b}^{2}+\omega^{2}(t) b^{2}+\frac{\omega_{0}^{2}}{b^{2}}\right)-\frac{1}{2} \hbar \omega(t),
$$

In the isentropic expansion stroke of an Otto cycle the non-adiabatic energy gives the dissipated work, that must vanish at $t_{f}$ for a STA process. Its time average has been proposed to quantify the cost to implement a quantum engine based on the harmonic oscillator [27].

The time-averaged non-adiabatic energy is

$$
\overline{E^{n a}}=\frac{\hbar \omega_{0}}{4 t_{f}} \int_{0}^{t_{f}}\left(\frac{\dot{b}^{2}}{\omega_{0}^{2}}+\frac{\omega^{2}(t) b^{2}}{\omega_{0}^{2}}+\frac{1}{b^{2}}-\frac{2 \omega(t)}{\omega_{0}}\right) d t .
$$

Using Ermakov's equation, partial integration and the conditions (4), we get a simpler form,

$$
\overline{E^{n a}}=\overline{E_{2}^{n a}} \equiv \frac{\hbar \omega_{0}}{2 t_{f}} \int_{0}^{t_{f}}\left(\frac{\dot{b}^{2}}{\omega_{0}^{2}}+\frac{1}{b^{2}}-\frac{\omega(t)}{\omega_{0}}\right) d t .
$$

where, again, we have introduced a special notation for the last integral, $\overline{E_{2}^{n a}}$, to emphasize that it becomes the time average of the non-adiabatic energy provided the boundary conditions (4) are satisfied. 


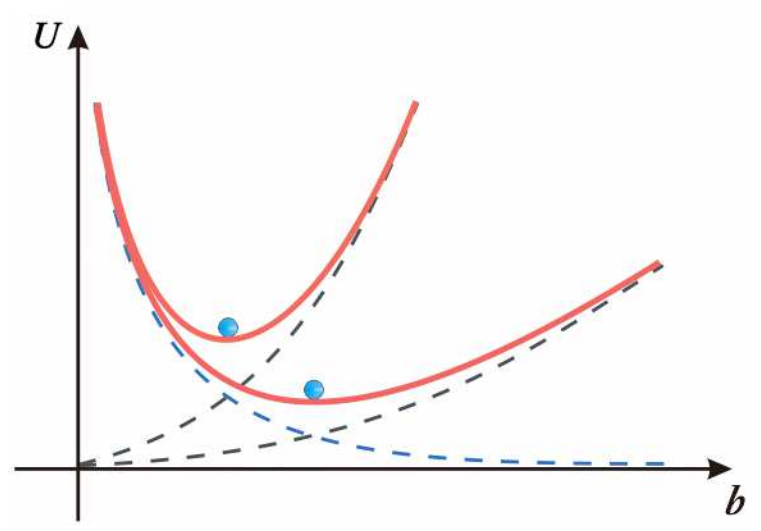

FIG. 2: (Color online) A classical particle (blue dot) moves along the bottom of an expanding potential $U(t)=$ $\left[\omega(t)^{2} b^{2}+\omega_{0}^{2} / b^{2}\right] / 2$ (red line), see text. The two parts of $U(t)$ are shown, $\omega(t)^{2} b^{2} / 2$ (blue dashed line), and $\omega_{0}^{2} /\left(2 b^{2}\right)$ (black dashed line).

\section{A. Lower bound for time-averaged non-adiabatic energy}

A useful analogy exists between the Ermakov equation and the dynamical equation of a fictitious (classical!) particle with mass $m=1$ and dimensionless "position" $b$ moving in a potential of the form [44]

$$
U(t)=\frac{1}{2}\left[\omega^{2}(t) b^{2}+\omega_{0}^{2} / b^{2}\right]
$$

with kinetic energy $\dot{b}^{2} / 2$. Newton's equation takes indeed the form of the Ermakov equation,

$$
\ddot{b}=\omega_{0}^{2} / b^{3}-\omega^{2}(t) b .
$$

This analogy provides useful intuition and will help us to find the lower bound for $\overline{E^{n a}}$. The corresponding (dimensionless) energy is

$$
\mathcal{H}=\frac{1}{2}\left(\dot{b}^{2}+\omega^{2}(t) b^{2}+\frac{\omega_{0}^{2}}{b^{2}}\right) .
$$

Up to a constant factor, it has the same form than the total energy (7), $E_{0}=\frac{\hbar}{2 \omega_{0}} \mathcal{H}$. Beware that the kinetic and potential energies of the classical particle do not correspond in general to the quantum counterparts in Eqs. (89). Specifically, the term $\omega_{0}^{2} /\left(2 b^{2}\right)$ is "kinetic" in the quantum scenario, and "potential" in the classical analogy.

Let us now calculate the excitation energy of the particle $E^{e x}$ measured from the potential minimum. To find the minimum we calculate $d U(t) / d b=0$, which leads to

$$
\omega(t)=\frac{\omega_{0}}{b^{2}(t)} .
$$

and $U_{\min }=\omega_{0} \omega$, so that

$$
E^{e x}=\frac{1}{2} \dot{b}^{2}+\frac{1}{2}\left(\omega^{2} b^{2}+\frac{\omega_{0}^{2}}{b^{2}}-2 \omega_{0} \omega\right) .
$$

This has exactly the same form than the non-adiabatic energy (31) up to the scaling factor, $E^{n a}=\frac{\hbar}{2 \omega_{0}} E^{e x}$. To complete the analogy, we consider that $\omega(t)$ changes from $\omega_{0}$ to $\omega_{f}$ and trajectories from the initial to the final points of the potential minimum, $b(0)=1$ to $b\left(t_{f}\right)=\gamma$.

Note that both the first term (kinetic energy) and the second one (potential energy measured from the minimum) are positive. The potential part of Eq. (38) can in fact be made zero if the particle moves all the time at the bottom of the potential, without ever being affected by a force, then Eq. (37) should hold and, substituted in Eq. (35) this gives the equation

$$
\ddot{b}=0
$$

with solution

$$
b=1+\frac{\gamma-1}{t_{f}} t,
$$

that satisfies the boundary conditions $b(0)=1$ and $b\left(t_{f}\right)=\gamma$. This linear $b$ also minimizes the time-averaged kinetic energy for these boundary conditions, as it can be seen from the Euler-Lagrange equation. However the particle trajectory described by Eq. (40) is not at rest at $t=0$ and $t_{f}$, so for the more restricted family of trajectories satisfying Eq. (4) it only provides a lower bound for $\overline{E^{e x}}$. Of course the same (scaled) lower bound is valid for the analogous quantum system, namely

$$
\overline{E_{L}^{n a}}=\frac{\hbar}{4 \omega_{0}}\left(\frac{\gamma-1}{t_{f}}\right)^{2} .
$$

When $\gamma \gg 1, \overline{E_{L}^{n a}} \approx \frac{\hbar}{4 \omega_{f} t_{f}^{2}}$, which is half the lower bound of the time-averaged energy for the ground dynamical mode if, in addition, $t_{f} \ll 1 / \sqrt{\omega_{0} \omega_{f}}$. Under these conditions the quantum kinetic energy is dominated by the first term in Eq. (8), which corresponds to the classical kinetic energy, and the virial theorem implies $\overline{K_{0}}=\overline{E_{0}} / 2$.

\section{B. Comparison of protocols}

The linear trajectory of $b(t)$ in Eq. (40) does not satisfy all the boundary conditions (4) and thus the protocol $\omega(t)=\omega_{0} / b^{2}$ based on it leads to excitation. Dirac impulses are not an option now, as the one at $t=0$ would have to be negative but $\omega^{2}<0$ is not allowed. We may instead complement the protocol with caps of durations $\tau_{L}$ and $\tau_{S}$ (for "launching" and "stopping" respectively) that connect the linear function with the proper boundary conditions, similarly to [8]. A hybrid protocol defined in this manner is

$$
b(t)= \begin{cases}\sum_{n=0}^{3} f_{n} s^{n}, & 0 \leq t \leq \tau_{L} \\ (\gamma-1) s+1, & \tau_{L} \leq t \leq t_{f}-\tau_{S}, \\ \sum_{n=0}^{3} g_{n} s^{n}, & t_{f}-\tau_{S} \leq t \leq t_{f}\end{cases}
$$




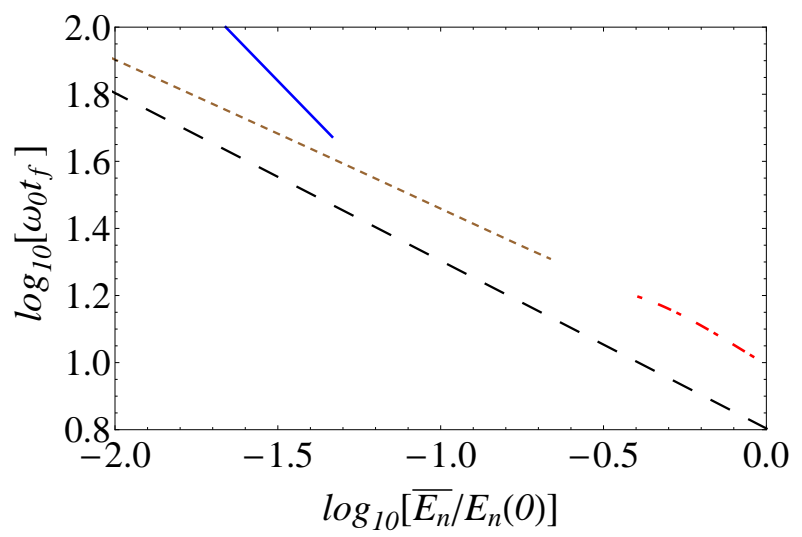

FIG. 3: (Color online) Dependence of $t_{f}$ on the time-averaged nonadiabatic energy for the hybrid protocol in Eq. (42) (dotted brown line), quintic polynomial protocol (solid blue line), bound in Eq. (41) (dashed black line), and bang-bang protocol with different bounds (dot-dashed red line). The parameters are $\omega_{0}=2500 \times 2 \pi \mathrm{Hz}$ and $\omega_{f}=25 \times 2 \pi \mathrm{Hz}$.

where $s=t / t_{f}$, the coefficients $f_{n}$ are found from the equations that match $b$ and $\dot{b}$ at 0 and $\tau_{L}$, and the $g_{n}$ from the matching at $t_{f}-\tau_{S}$ and $t_{f}$. It can be proved that in the cubic interpolation $b>0$. The cap durations $\tau_{L, S}$ can be adjusted by a subroutine that minimizes $\overline{E^{n a}}$ with the constraint $\omega \geq 0$. Due to the constraint the cap times cannot be made zero, so, unlike Sec. IIB, the bound is not reached. The caps can only be constructed in this way until a minimal $t_{f}$ for which imaginary frequencies appear.

We also consider bang-bang protocols that provide a meaningful non-adiabatic energy with $\omega_{1}=0$, and $\omega_{2}=$ $\omega_{0} \beta, \beta>0$, see the Appendix, with

$$
\begin{aligned}
& t_{1}=\frac{1}{\omega_{0}} \sqrt{\frac{\left(\gamma^{2}-1\right)\left(\gamma^{2} \beta^{2}-1\right)}{\gamma^{2} \beta^{2}}}, \\
& t_{2}=\frac{1}{\omega_{0} \beta} \arcsin \left[\sqrt{\frac{\gamma^{2}-1}{\beta^{2} \gamma^{4}-1}}\right] .
\end{aligned}
$$

Fig. 3 shows the scaling of $t_{f}$ and the time-averaged non-adiabatic energy. Note that there is some complementarity among the different protocols, which are applicable in different domains. Whereas the bang-bang protocol only satisfies the boundary conditions in a smalltime, high-energy regime, the quintic polynomial (6) or hybrid protocols apply rather in a small-energy, lowenergy scenario.

\section{POWER}

We finally consider the instantaneous power during the STA process,

$$
P_{n}(t) \equiv \frac{d E_{n}}{d t}=k_{n} \dot{\mathcal{K}} b^{2}
$$

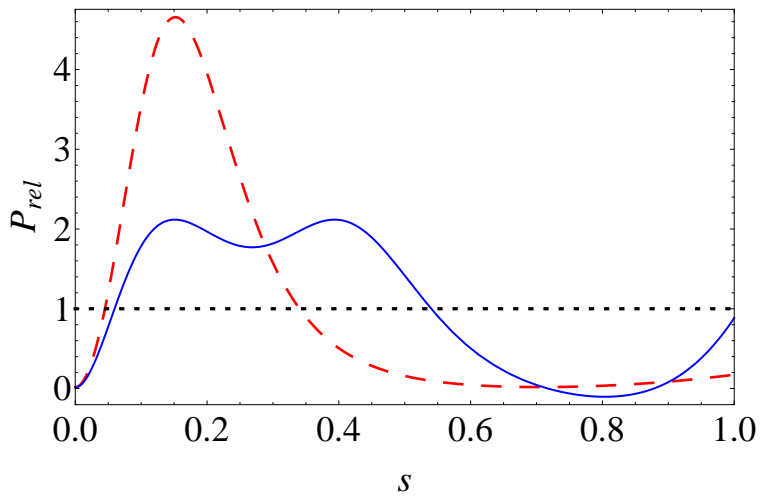

FIG. 4: (Color online) Relative power versus $s=t / t_{f}$ for the polynomial (6) (dashed red line) and for the polynomial (50) (solid blue) with $c_{3}=78.5088, c_{4}=-459.7638$. Parameters: $\omega_{0}=2500 \times 2 \pi \mathrm{Hz}, \omega_{f}=25 \times 2 \pi \mathrm{Hz}$, and $t_{f}=8 \mathrm{~ms}$.

where we have used Eq. (7) and defined $k_{n} \equiv \frac{\hbar(2 n+1)}{4 \omega_{0}}$ and $\mathcal{K}(t) \equiv \omega^{2}$. This is a remarkably simple expression for $P_{n}$. (Defining the time-independent operator $v$ by $V=\mathcal{K}(t) v$, and using Eq. (9), it may also be written as $\dot{\mathcal{K}}\langle v\rangle$ which is the form given in [45].) For any STA process its integral is

$$
\int_{0}^{t_{f}} \frac{d E_{n}}{d t} d t=E_{n}\left(t_{f}\right)-E_{n}(0)=\left(n+\frac{1}{2}\right) \hbar\left(\omega_{f}-\omega_{0}\right) .
$$

As an example of the possible use of power for shortcut design, suppose that we are interested in minimizing the power peak (maximum) of $\left|P_{n}\right|$, distributing the power homogeneously from 0 to $t_{f}$ to facilitate the power extraction. We then set

$$
P_{n}=k_{n} \dot{\mathcal{K}} b^{2}=C_{n},
$$

where $C_{n}$ is a constant adjusted to satisfy Eq. (46), namely

$$
C_{n}=(n+1 / 2) \hbar\left(\omega_{f}-\omega_{0}\right) / t_{f},
$$

note that $C_{n} / k_{n}$ does not depend on $n$, so that the relative power $P_{\text {rel }} \equiv P_{n} / C_{n}$ does not depend on $n$. According to the mean value theorem, 1 is a lower bound for the maximum of $P_{r e l}$ in an arbitrary STA expansion process. $\mathcal{K}$ and $b$ must satisfy the Ermakov equation (2) so Eq. (47) becomes

$$
b \dddot{b}-\ddot{b} \dot{b}+4 \frac{\omega_{0}^{2}}{b^{3}} \dot{b}=2\left(\omega_{0}-\omega_{f}\right) \frac{\omega_{0}}{t_{f}} .
$$

This is a third order equation. To avoid a sudden jump in $\omega(t)$ at time $t=0$, and thus a Dirac delta in the power, we must impose $\ddot{b}(0)=0$ so in fact, with $b(0)=1$ and $\dot{b}(0)=0$ we already fix the three possible constants at time $t=0$. The numerical solution may satisfy $b\left(t_{f}\right)=\gamma$ for a specific $t_{f}$ but the conditions $\dot{b}\left(t_{f}\right)=\ddot{b}\left(t_{f}\right)=0$ fail in general. We may thus resort to more modest objectives. 
As an example of many possible strategies, in Fig. 4 we plot $P_{\text {rel }}$ for the fifth order polynomial (6) and for a polynomial that satisfies the boundary conditions (4) and (5) with two free parameters that are chosen to minimize numerically the maximum of $P_{r e l}$,

$$
\begin{aligned}
& b(t)=1+c_{3} s^{3}+c_{4} s^{4}-\left(21+6 c_{3}+3 c_{4}-21 \gamma\right) s^{5} \\
& +\left(35+8 c_{3}+3 c_{4}-35 \gamma\right) s^{6}-\left(15+3 c_{3}+c_{4}-15 \gamma\right) s^{7}
\end{aligned}
$$

where $s=t / t_{f}$.

\section{DISCUSSION}

Different transient energies in shortcuts to adiabatic harmonic expansions for a single particle have been studied, as well as the relations with the process time. This is important to establish operational limits, which are different from the naive application of a time-energy uncertainty relation. General bounds are provided, and protocols that realize or approach them have been discussed. The specific experimental conditions, i.e. the actual realization of the system and the potential by different interactions (optical, magnetic, electrostatic), will determine their realizability, since optimal protocols often imply Dirac deltas (e.g. in the potential, or in the power).

The virial theorem for time averaged energies emerges as an important relation for STA processes. We have given examples where it is instrumental in providing bounds and interpreting the results. Further relations among various energies and their applications will be discussed elsewhere, extending the theorem to other operations and potentials.

The power in STA has been also brought to the fore. It provides a further criterion to choose among different shortcuts. Even though its time integral is invariant for all STA, the maximal values could be minimized.

\section{Acknowledgments}

This work was partially supported by the NSFC (61176118 and 11474193), the Shanghai Shuguang Program (14SG35), the Shanghai Pujiang Program (13PJ1403000), the program for Eastern Scholar, the Specialized Research Fund for the Doctoral Program of Higher Education (Grant No. 2013310811003), the Basque Government (Grant IT472-10), MINECO (Grant FIS2012-36673-C03-01), and the program UFI 11/55 of $\mathrm{UPV} / \mathrm{EHU}$.

\section{Appendix A: Bang-bang protocols}

A "bang-bang" protocol is composed by time segments with constant frequency so that the energy rate transfer (power) from or to the system consists of Dirac deltas at the switching times. For applications and discussion of their relation to Optimal Control Theory see [4, 7, 11, 12, 14. Typically the frequency is chosen at the extreme values allowed to achieve optimal results.

We consider here processes with two intermediate steps of the form (more switching times may be required to find the true minimal time in general [11])

$$
\omega(t)=\left\{\begin{array}{l}
\omega_{0}, \quad t=0 \\
i \omega_{1}, \quad 0<t<t_{1} \\
\omega_{2}, \quad t_{1}<t<t_{1}+t_{2} \\
\omega_{f}, \quad t=t_{f}=t_{1}+t_{2}
\end{array}\right.
$$

where $\omega_{1} \geq 0$ and $\omega_{2}>0$ are real constants, and $t_{1}$ and $t_{2}$ are the durations of each part. For $0<t<t_{1}$, the solution of the Ermakov equation satisfying $b(0)=1$, and $\dot{b}(0)=0$ is

$$
b(t)=\sqrt{1+\frac{\omega_{0}^{2}+\omega_{1}^{2}}{\omega_{1}^{2}} \sinh ^{2}\left(\omega_{1} t\right)},
$$

and for $t_{1} \leq t \leq t_{f}$, the one that satisfies $b\left(t_{f}\right)=\gamma$, $\dot{b}\left(t_{f}\right)=0$ is

$$
b(t)=\sqrt{\gamma^{2}+\frac{\omega_{0}^{2}-\gamma^{4} \omega_{2}^{2}}{\gamma^{2} \omega_{2}^{2}} \sin ^{2}\left[\omega_{2}\left(t_{f}-t\right)\right]} .
$$

The matching conditions for $b\left(t_{1}\right)$ and $\dot{b}\left(t_{1}\right)$ determine

$$
\begin{aligned}
& t_{1}=\frac{1}{\omega_{1}} \operatorname{arcsinh} \sqrt{\frac{\omega_{1}^{2}\left(\gamma^{2}-1\right)\left(\gamma^{2} \omega_{2}^{2}-\omega_{0}^{2}\right)}{\gamma^{2}\left(\omega_{2}^{2}+\omega_{1}^{2}\right)\left(\omega_{0}^{2}+\omega_{1}^{2}\right)}}, \\
& t_{2}=\frac{1}{\omega_{2}} \arcsin \sqrt{\frac{\omega_{2}^{2}\left(\gamma^{2}-1\right)\left(\gamma^{2} \omega_{1}^{2}+\omega_{0}^{2}\right)}{\left(\omega_{2}^{2}+\omega_{1}^{2}\right)\left(\gamma^{4} \omega_{2}^{2}-\omega_{0}^{2}\right)}} .
\end{aligned}
$$

There is an upper bound for $t_{f}$. According to Eq. (A4), $\omega_{2} \geq \sqrt{\omega_{0} \omega_{f}}$ must be satisfied to make $t_{1} \geq 0$. With this condition, $t_{2}$ will be positive. At $\omega_{2}=\sqrt{\omega_{0} \omega_{f}}, t_{1}=$ 0 , which gives the upper upper bound for $t_{f}$ and the corresponding minimal time-averaged energy,

$$
\begin{aligned}
t_{f}^{\max } & =\frac{\pi}{2 \sqrt{\omega_{0} \omega_{f}}}, \\
\overline{E_{n}^{\min }} & =(2 n+1) \hbar \frac{\omega_{0}+\omega_{f}}{4} .
\end{aligned}
$$

To calculate $\overline{E_{n}}$, we use the fact that the total energy remains constant during the constant-frequency intervals. It can be calculated with Eqs. (8) and (9), at $t=0^{+}$for the first segment and at $t=t_{f}^{-}$for the second segment,

$$
E_{n}=\frac{(n+1 / 2) \hbar}{2} \begin{cases}\left(\omega_{0}^{2}-\omega_{1}^{2}\right) / \omega_{0}, & 0<t<t_{1} \\ \left(\omega_{f}^{2}+\omega_{2}^{2}\right) / \omega_{f}, & t_{1}<t<t_{f}\end{cases}
$$

If $\omega_{1}=\omega_{2} \gg \omega_{0}$ with $\gamma \gg 1, t_{f}$ approaches zero, and the corresponding time-averaged energy is

$$
\overline{E_{n}} \approx \frac{(2 n+1) \pi \hbar}{16 \omega_{f} t_{f}^{2}} \ln [2 \gamma]
$$


This is of course larger than the asymptotic value of lower bound for the time-averaged energy, $\overline{E_{n L}} \approx \frac{(2 n+1) \hbar}{2 \omega_{f} t_{f}^{2}}$.

If we set $\omega_{1}=0, \omega_{2} \gg \omega_{0}$, and $\gamma \gg 1$ then

$$
\begin{gathered}
t_{f} \approx t_{1} \approx \frac{1}{\sqrt{\omega_{0} \omega_{f}}}, \\
\overline{E_{n}} \approx(n+1 / 2) \hbar \omega_{0} .
\end{gathered}
$$

or, making use of the relation between $t_{f}$ and $\omega_{0}$ in Eq. A8 we find again the bound value, namely $\overline{E_{n}} \approx$ $\frac{(2 n+1) \hbar}{2 \omega_{f} t_{f}^{2}}$. We may interpret this result in view of the virial theorem. Under these conditions most of the protocol time is just a free expansion ended up by a short segment with a strong potential. The frequency switch at time zero suddenly turns off the initial harmonic potential so that half the initial energy vanishes, and for most of the time the energy (purely kinetic) is $E_{n}(0) / 2$. Thus $\overline{K_{n}} \approx E_{n}(0) / 2$, and according to the virial theorem the last segment must be such that $\overline{V_{n}}=E_{n}(0) / 2$ as well. That explains why $\overline{E_{n}} \approx E_{n}(0)$.
[1] E. Torrontegui, S. Ibáñez, S. Martínez-Garaot, M. Modugno, A. del Campo, D. Guéry-Odelin, A. Ruschhaupt, X. Chen, J. G. Muga, Adv. At. Mol. Opt. Phys. 62, 117 (2013).

[2] J. F. Schaff, X. L. Song, P. Vignolo, and G. Labeyrie, Phys. Rev. A 82, 033430 (2010); Phys. Rev. A 83, 059911(E) (2011).

[3] J. F. Schaff, X. L. Song, P. Capuzzi, P. Vignolo, and G. Labeyrie, EPL, 93, 23001 (2011).

[4] P. Salamon, K. H. Hoffmann, Y. Rezek, and R. Kosloff, Phys. Chem. Chem. Phys. 11, 1027 (2009).

[5] J. G. Muga, X. Chen, A. Ruschhaupt, and D. GuéryOdelin, J. Phys. B 42, 241001 (2009).

[6] T. Schmiedl, E. Dieterich, P.-S. Dieterich and U. Seifert, J. Stat. Mech. P07013 (2009).

[7] X. Chen, A. Ruschhaupt, S. Schmidt, A. del Campo, D. Guéry-Odelin, and J. G. Muga, Phys. Rev. Lett. 104, 063002 (2010).

[8] X. Chen, and J. G. Muga, Phys. Rev. A 82, 053403 (2010).

[9] J. G. Muga, X. Chen, S. Ibáñez, I. Lizuain, and A. Ruschhaupt, J. Phys. B: At. Mol. Opt. Phys. 43, 085509 (2010).

[10] S. Masuda and K. Nakamura, Proc. R. Soc. A 466, 1135 (2010).

[11] D. Stefanatos, J. Ruths, and Jr-Shin Li, Phys. Rev. A 82, 063422 (2010).

[12] D. Stefanatos and J.-S. Li, 2012 American Control Conference, Montreal, Canada, pp. 5061-5066, (2012); arXiv:1103.1665.

[13] D. Stefanatos and J.-S. Li, SIAM J. Cont. Opt. 49, 2440 (2011).

[14] K. H. Hoffmann, P. Salamon, Y. Rezek and R. Kosloff, EPL, 96, 60015 (2011).

[15] Y. Li, L. A. Wu, and Z. D. Wang, Phys. Rev. A 83, 043804 (2011).

[16] A. del Campo, Phys. Rev. A 84, 031606(R) (2011).

[17] J. F. Schaff, P. Capuzzi, G. Labeyrie, and P. Vignolo, New. J. Phys. 13, 113017 (2011).

[18] S. Choi, R. Onofrio, and B. Sundaram, Phys. Rev. A 84, 051601(R) (2011).

[19] E. Torrontegui, S. Martínez-Garaot, A. Ruschhaupt, and J. G. Muga, Phys. Rev. A 86, 013601 (2012).

[20] S. Choi, R. Onofrio, and B. Sundaram, Phys. Rev. A 86, 043436 (2012).
[21] J.-Q. Zhang, L. Yong, M. Feng, J. Phys.: Cond. Matt. 25, 142201 (2013).

[22] D. Stefanatos and J.-S. Li, Phys. Rev. A 86, 063602 (2012).

[23] B. Juliá-Díaz, E. Torrontegui, J. Martorell, J. G. Muga, and A. Polls, Phys. Rev. A 86, 063623 (2012).

[24] E. Torrontegui, S. Martínez-Garaot, A. Ruschhaupt, and J. G. Muga, Phys. Rev. A 86, 013601 (2012).

[25] A. Yuste, B. Juliá-Díaz, E. Torrontegui, J. Martorell, J. G. Muga, and A. Polls, Phys. Rev. A 88, 043647 (2013).

[26] D. Guéry-Odelin, J. G. Muga, M. J. Ruiz-Montero, and E. Trizac, Phys. Rev. Lett. 112, 180602 (2014).

[27] A. del Campo, J. Goold, and M. Paternostro, Scientific Reports 4, 6208 (2014).

[28] J. W. Deng, Q. H. Wang, Z. H. Liu, P. Hänggi, and J. B. Gong, Phys. Rev. E 88, 062122 (2013).

[29] C. Jarzynski, Phys. Rev. A 88, 040101(R) (2013).

[30] M. Palmero, S. Martínez-Garaot, J. Alonso, J. P. Home, J. G. Muga, Phys. Rev. A, accepted; arXiv:1502.00998

[31] E. Torrontegui, X. Chen, M. Modugno, A. Ruschhaupt, D. Guéry-Odelin, and J. G. Muga, Phys. Rev. A 85, 033605 (2012).

[32] X.-J. Lu, X. Chen, J. Alonso, and J. G. Muga, Phys. Rev. A 89, 023627 (2014).

[33] O. Abah, J. Rossnagel, G. Jacob, S. Deffner, F. SchmidtKaler, K. Singer, and E. Lutz, Phys. Rev. Lett. 109, 203006 (2012).

[34] H. R. Lewis and W. B. Riesenfeld, J. Math. Phys. 10, 1458 (1969).

[35] E. Torrontegui, S. Ibáñez, X. Chen, A. Ruschhaupt, D. Guéry-Odelin, J. G. Muga, Phys. Rev. A 83, 013415 (2011).

[36] V. Fock, Zeitschrift für Physik A 63855 (1930).

[37] J. C. Slater, J. Chem. Phys. 1, 687 (1933).

[38] E. Merzbacher, Quantum Mechanics, (Wiley, New York, 1961), p. 168.

[39] F. S. Crawford, Am. J. Phys. 57, 555 (1989).

[40] T. C. Ernest Ma, Am. J. Phys. 59, 476 (1991).

[41] J. G. Muga, Am. J. Phys. 62, 183 (1994).

[42] D. H. Kobe, Am. J. Phys. 63, 174 (1995).

[43] A. E. Allahverdyan, R. S. Gracià, and Th. M. Nieuwenhuizen, Phys. Rev. E 71, 046106 (2005).

[44] A. Davydov, Int. J. Theor. Phys. 50, 1451 (2011).

[45] R. Kosloff, J. Chem. Phys. 80, 1625 (1984). 